\title{
BIRDS OF A EUCALYPTUS WOODLOT IN INTERIOR SÃO PAULO
}

\author{
WILLIS, E. O. \\ Departamento de Zoologia, UNESP, CEP13506-900, Rio Claro, SP, Brazil \\ Correspondence to: Edwin O. Willis, Departamento de Zoologia, UNESP, CEP13506-900, \\ Rio Claro, SP, Brazil, e-mail: ewillis@rc.unesp.br \\ Received April 10, 2001 - Accepted April 29, 2002 - Distributed February 28, 2003
}

\begin{abstract}
Some 255 birds were recorded between 1982-2001 in and near a 2314-ha "Horto" of old eucalyptus plantations with native understory and a lake, near Rio Claro, in central São Paulo, Brazil. This is close to the 263 recorded in and around a ten-times smaller nearby 230-ha woodlot of semideciduous forest. Different species were 44, for a total of 307 in both areas. One hundred and fifty nonvagrant forest and border species were recorded in 1982-86, a number close to the 152 in the small native woodlot. With dry years and logging of plots in 1985-93, 21 of the 150 species were lost, 42 species decreased in numbers, 49 were stable, 19 increased (15 being border species), and 5 entered (one of dry forest and 4 of borders), so 129 species remained in 1996-2001 compared to 133 in the native woodlot. Open-area birds were 33, versus 50 in better-checked grassy swales in sugar cane near the natural woodlot, for a total of 53. Several species, like some border ones, did not enter the open but isolated and mowed interior lake area, or took years to do so. Water and marsh birds were 46 versus 40 in smaller creeks and ponds near the natural woodlot (total, 55) but many were migrants or infrequent visitors using distant areas, and perhaps should be counted as 0.1-0.9 "local species" rather than "1" species. Use of this more accurate method would reduce waterbird totals by 14 "species" in the Horto and by 11 around the native woodlot. I also recommend longer censusing at the edges in large woodlots or many edge species will be recorded only in small fragments of habitat. Several species increased and others decreased with occasional cat-tail and water-lily cleanups at the lake. A forested corridor between the Horto and natural woodlot is recommended, with old eucalyptus left to provide flowers for hummingbirds.
\end{abstract}

Key words: birds, censuses, corridors, eucalyptus, fragmentation, São Paulo.

\section{RESUMO}

\section{Aves de uma mata de eucaliptos no interior de São Paulo}

Cerca de 255 espécies de aves foram registradas entre 1982-2001 dentro e próximo do Horto Florestal, em 2314 ha de plantações antigas de eucaliptos e sub-bosque nativo com um lago, próximo a Rio Claro, no centro do Estado de São Paulo, Brasil. Esse registro é próximo das 263 espécies registradas dentro e próximo de uma mata natural dez vezes menor, com 230 ha. As espécies diferentes foram 44, para um total de 307 em ambas as áreas. Cento e cinqüenta espécies não-vagantes de mata e borda foram registradas em 1982-86, assim como 152 na mata nativa pequena. Com os anos secos e a retirada de madeira em 1985-93, 21 das 150 espécies desapareceram, 42 espécies diminuíram em números, 40 permaneceram estáveis, 19 aumentaram ( 15 sendo espécies de borda) e 5 surgiram (uma de mata seca e 4 de borda), portanto, 129 espécies permaneceram em 1996-2001 se comparado com 133 na mata nativa. As aves de áreas abertas foram 33 , versus $50 \mathrm{em}$ áreas de canaviais junto à área florestada, para um total de 53. Várias espécies, assim como algumas de borda, não entraram em áreas abertas do lago, isoladas e capinadas, ou levaram vários anos para entrar. As aves aquáticas e de brejo foram 46 versus 40 em pequenos riachos e lagos próximos à área natural (total 55), mas muitas eram 
migrantes ou visitantes infreqüentes usando áreas distantes, e talvez devessem ser contadas como 0,10,9 "espécie local", em vez de " 1 " espécie. O uso deste método mais refinado reduziria os totais para aves aquáticas de 14 "espécies" no Horto e de 11 nos arredores da área nativa. Também, recomendo efetuar o censo por longos períodos nas margens de grandes áreas, ou muitas espécies de borda serão registradas somente em pequenos fragmentos de habitat. Várias espécies aumentaram e outras diminuíram com as ocasionais limpezas do lago e retiradas de taboais e lírios-de-água. Um corredor de mata entre o Horto e a área natural é recomendado, com os velhos eucaliptos se encarregando de proporcionar flores para os beija-flores.

Palavras-chave: aves, censos, corredores, eucaliptos, fragmentação, São Paulo.

\section{INTRODUCTION}

Native semideciduous woods and cerrados have generally been destroyed on the relatively flat and fertile interior plateau of São Paulo State, southeastern Brazil. Dense cities next to croplands (cane, orange, and eucalyptus) remind one of the very productive "Kyushu World" of southern Japan, though cattle raised indoors and rice fields by tiny yards have not yet reached Brazil. Near the town of Rio Claro, between the Tietê and Mogi-Guaçu Rivers at about $650 \mathrm{~m}$ elevation in the center of São Paulo State, Willis \& Oniki (2002a) investigated birds of the Fazenda São José 230-ha semideciduous woodlot, plus nearby cane fields and creeks. Earlier studies checked birds of smaller to large tracts off to the south (Willis, 1979; Aleixo \& Vielliard, 1995; Magalhães, 1999) and northwest (Motta-Júnior, 1990). Here I report birds of a much larger Rio Claro seminatural area: a partly abandoned eucalyptus woodlot. Machado \& Lamas (1996) briefly studied birds of a similarly large eucalyptus area in Minas Gerais.

South just a few kilometers across cane fields from the São José remnant woodlot, and just east of the rather dirty Ribeirão Claro linking the two, at the east edge of town (by the campus of the Universidade Estadual Paulista), there is a 2314ha eucalyptus woodlot called the "Horto Florestal". A normal eucalyptus zone would be nearly birdless, for it would be cut every 7-10 years, not have flowers, would be cleared out underneath and have no ponds or semi-open village ("Area IV" of Machado \& Lamas, 1996; Aracruz Florestal areas in Espírito Santo, pers. obs.). However, the Horto, which was run by a state railroad company (FEPASA) for railroad ties, was where eucalyptus research started in Brazil on a coffee ranch (there is still some coffee in the understory). When FEPASA was sold to a private company in 1998, dozens of other FEPASA eucalyptus groves were given away without being checked for rare species to the landless poor or "sem-terra", for them to cut wood and plant crops, but the Horto and one coastal woodlot went to the Instituto Florestal (IF) of São Paulo State.

The Horto had large eucalyptus all over, often planted from 1910 to 1950 . Dense vine and bush understories were allowed to grow, plus patches of secondary and creek woods. A farm pond (Córrego Ibitinga), botanic gardens, nearby lawns and a village (next to a eucalyptus museum) occupied a few inner hectares near the west edge. Both FEPASA and IF had their headquarters at a few other ranch houses by the Ribeirão Claro, while upcreek a watertreatment plant provided city water and there were swamp woods battered by illegal fishermen. Dirt roads crossed, and small ones ran between plantation blocks $500 \mathrm{~m}$ or so on a side.

Mitchell (1957) mentions birds at the pond, and two bachelor's theses (Ferrari, 1985; Antunes, 1996) report birds there; another master's thesis (Antunes, 2000) reports birds at eucalyptus flowers. The scenic pond and garden zone are popular for Sunday picnics; fruiting palms and trees are planted about, and the lawns are mowed irregularly. Cattails and willows at the pond edge, or floating water lilies, are cleared out now and then. Otherwise, cattails would take over as in a similar marsh by $\mathrm{Fa}-$ zenda São José. However, eroded sediment in the pond (the side creek comes through the woodlot from cane fields off to the east) has not been dredged, unlike the deep "Lago Azul" in the city, where people pedal around in boats and have removed all the natural vegetation. In the 1980 s, roads through the eucalyptus started to be used by joggers, while capybaras at the lake were hunted or eliminated (like certain birds) by clean-up activities. In 2001, a physical exercise route was set up. 
Cutting of the $500 \mathrm{~m}$ long eucalyptus tracts suddenly resumed in 1985, notably along jogging routes I had been censusing. One tract was removed, two more in 1989 (with weeds cut and burned in the 1985 tract), another in 1990, which was replanted with eucalyptus saplings. An uncensused tract at the southeast corner was also razed (bushes grew back). A pasture tract near the east edge was also replanted, so that dense stands of slender eucalyptus saplings persist in 5 tracts until today. Perhaps joggers complained of the hot sun, for cutting of six other tall-forest tracts in 1991-94 was partial, leaving scattered tall eucalyptus but destroying nearly all the native understory (which grew back to some extent). Only 3 of 14 tracts along the study route continued untouched, plus 3-4 tracts near the lake where native understory grew under teak or other abandoned trees. Only a few saplings were removed later, with some road clearing activity or firebreaks (the grass under the pasture sapling tract burned in mid-1997). Cows were introduced to eat grass under sapling tracts in 1991, 1992, and 1995-96, plus a few horses in 1999, but affected the understory little in the 9 other partially cut or uncut tracts studied.

\section{METHODS}

Morning censuses were transects, with total numbers of birds and hours registered per day; night hours are registered separately (10-30 min. at dawn, along the road east across the Horto). The Horto lake (at $590 \mathrm{~m}$ and $22^{\circ} 25^{\prime} \mathrm{S}, 47^{\circ} 31^{\prime} \mathrm{W}$ ) was nearly always part of the census; it was the main area visited for ornithology classes (1 day in 1985 and 99; 2 in 1986, 90, 93, 94, and 97; 3 in 1987; 4 in 1989 and 1991; 5 in 2000) and for some other visits ( 7 in 1982; 3 in 1987; and 1 each in 1983, $85,94,95)$. From there, I walked east on other visits to an old corral in the woods and then via a spider-filled lane (now cleared for the exercise route) south to $500 \mathrm{~m}$ on the jogging road, following this east to $1200 \mathrm{~m}$, and north across the hilltop to the Ibitinga Creek bridge at 3300 $\mathrm{m}$. I then walked back to $2100 \mathrm{~m}$ on the road, west some $400 \mathrm{~m}, 800 \mathrm{~m}$ south off the hill, down to the corral, and out at the botanic garden and lake. From 1997 on, one had to park $500 \mathrm{~m}$ before the lake at the village entrance near an old church where I often crossed the creek on a wooden bridge on arrival, returning via the village. Three censuses in 1982 (13 Aug., 30 Sept., 29 Oct.) were from the nearby campus along the Ribeirão Claro at the northwest edge of the Horto, in marshes and swamps along fishing trails. Three short visits were at the northeast corner of the Horto, in eucalyptus areas (3, 8, and 22 Aug. 1982). Besides the normal routes, I sometimes added 0.5-1.5 hour at dawn along the main road east, especially at the east cane-field edge (5 Dec. 84, 7 in 1987, 2 in 88, 6 in 89, 2 in 91, 6 in 93,5 in 94,2 in 95,1 in 96,2 in 97,4 in 98 , 3 in 99,6 in 00 and 1 in 01 ).

\section{RESULTS}

Some 255 species were recorded in and next to the Horto (Table 1), including 44 species ("+") not recorded among 263 in and near the São José forest (Willis \& Oniki, 2002a). Thus, 307 species are recorded for the two areas, including species in the intervening sugar cane fields and marshy swales. Asio stygius is recorded in the UNESP plaza (Willis and others), and Antunes (2000) recorded Hylocharis cyanus 26/7/98 (winter) at eucalyptus flowers in the Horto. He also recorded (pers. comm.) two rare birds that occur in São José (Brotogeris chiriri and Certhiaxis vulpina) plus wandering Mycteria americana, a new species for Rio Claro. This is considerably more species than the mere 150 or so on the similarly sized campus of USP in the city of São Paulo (Höfling \& Camargo, 1993), but less than the 354 recorded in the main woodlot and nearby open or reservoir areas at Fazenda Barreiro Rico (Magalhães, 1999, plus 3 species recently, A. Antunes and Willis).

As in Willis \& Oniki, 2002a, many species here were not birds of the woodlot or its borders. In Table 1, some 33 species were "A" birds of open areas (plus two of 11 vagrant species), while 46 nonvagrants (plus 1 vagrant) were marsh or pond species ("C"). Subtracting these species and 11 vagrants, 165 species were forest and border birds (the latter, "B", were 68 species, plus 2 vagrants). This is like the 163 forest and border species recorded in the much smaller São José natural woodlot, where some 40 species were "C", 50 were "A", and 60 nonvagrants (plus 5 of 10 vagrants) were "B". Forest-interior nonvagrant species were thus only 97 in the Horto and 103 in the much smaller but seminatural São José woodlot. Motta-Júnior (1990) and Machado \& Lamas (1996) reported fewer species in their eucalyptus areas, but their studies were brief. 
TABLE 1

Birds of the Rio Claro Horto, 1982-2001.

\begin{tabular}{|c|c|c|c|c|}
\hline Species & 1 & 2 & 3 & 4 \\
\hline Crypturellus parvirostris - A & 39 & 28 & 41 & 12 \\
\hline Crypturellus tataupa & 62 & 28 & 13 & - \\
\hline Nothura maculosa - A & 1 & - & 2 & 1 \\
\hline Dendrocygna viduata $-\mathrm{C}$ & 386 & 597 & 422 & 875 \\
\hline Dendrocygna autumnalis - C+ & - & - & - & 2 \\
\hline Cairina moschata - C & 30 & 43 & 201 & 386 \\
\hline Amazonetta brasiliensis - $\mathrm{C}$ & 341 & 420 & 314 & 128 \\
\hline Nomonyx dominicus $-\mathrm{C}$ & 72 & 110 & 43 & 31 \\
\hline Phalocrocorax brasilianus $-\mathrm{C}+$ & - & - & 1 & 2 \\
\hline Anhinga anhinga $-\mathrm{C}+$ & - & - & - & 10 \\
\hline Tachybaptus dominicus - C & 14 & 5 & 1 & 8 \\
\hline Podilymbus podiceps - C+ & 16 & 41 & 65 & 32 \\
\hline Ardea alba $-\mathrm{C}$ & 1 & 11 & 131 & 22 \\
\hline Egretta thula - C & - & 21 & 151 & 3 \\
\hline Bubulcus ibis - A & - & - & - & 1 \\
\hline Nycticorax nycticorax $-\mathrm{C}+$ & - & - & 5 & 1 \\
\hline Pilherodius pileatus - C & 3 & 19 & 13 & - \\
\hline Syrigma sibilatrix $-\mathrm{A}$ & 7 & 19 & 39 & 30 \\
\hline Butorides striatus - C & 94 & 95 & 64 & 17 \\
\hline Tigrisoma lineatum - C+ & 2 & 11 & 19 & 4 \\
\hline Botaurus pinnatus $-\mathrm{C}+$ & 2 & - & - & - \\
\hline Mesembrinibis cayennensis - C & - & 2 & 32 & 27 \\
\hline Platalea ajaja - CV+ & - & - & 1 & - \\
\hline Aramus guarauna - $\mathrm{C}+$ & 106 & 102 & 60 & 22 \\
\hline Aramides cajanea - $\mathrm{C}$ & 17 & 23 & 27 & 7 \\
\hline Aramides saracura $-\mathrm{C}$ & 136 & 136 & 88 & 22 \\
\hline Amaurolimnas concolor - C+ & 2 & - & - & - \\
\hline Rallus nigricans $-\mathrm{C}$ & 103 & 93 & 56 & 21 \\
\hline Laterallus melanophaius - C & 37 & - & - & 4 \\
\hline Gallinula chloropus - C & 750 & 1013 & 1470 & 1027 \\
\hline Porphyrula martinica $-\mathrm{C}+$ & 297 & 259 & 1 & 4 \\
\hline Cariama cristata $-\mathrm{A}$ & 9 & 17 & 22 & 12 \\
\hline Jacana jacana - C & 532 & 637 & 601 & 145 \\
\hline Vanellus chilensis - A & 47 & 103 & 126 & 119 \\
\hline Tringa solitaria $-\mathrm{CN}$ & - & - & 30 & 10 \\
\hline Tringa flavipes - $\mathrm{Cn}+$ & - & - & 2 & - \\
\hline Tringa melanoleuca $-\mathrm{Cn}+$ & 1 & - & - & - \\
\hline
\end{tabular}


TABLE 1 (Continued)

\begin{tabular}{|c|c|c|c|c|}
\hline Species & 1 & 2 & 3 & 4 \\
\hline Cathartes aura & 6 & 4 & 1 & - \\
\hline Coragyps atratus - B & 221 & 299 & 276 & 110 \\
\hline Gampsonyx swainsoni - B+ & (2) & - & 1 & - \\
\hline Rostrhamus sociabilis - C+ & - & 27 & 39 & 16 \\
\hline Ictinia plumbea - BT+ & 26 & 42 & 35 & 38 \\
\hline Leptodon cayanensis & 18 & 17 & 9 & 1 \\
\hline Chondrohierax uncinatus $-\mathrm{s}+$ & - & - & 1 & 2 \\
\hline Harpagus diodon $-\mathrm{s}$ & - & 4 & 3 & 2 \\
\hline Accipiter striatus - B & 7 & 5 & 1 & 7 \\
\hline Buteo magnirostris - $\mathrm{B}$ & 127 & 129 & 150 & 55 \\
\hline Buteo brachyurus - B & 4 & 5 & - & - \\
\hline Spizaetus tyrannus - V+ & 1 & - & - & - \\
\hline Caracara plancus - B & 7 & 15 & 34 & 57 \\
\hline Milvago chimachima - A & 20 & 31 & 54 & 31 \\
\hline Herpetotheres cachinnans - B & 9 & 9 & 12 & 7 \\
\hline Micrastur semitorquatus & 2 & 3 & - & 1 \\
\hline Falco femoralis - A & - & - & - & 1 \\
\hline Falco sparverius - A & - & - & 7 & 3 \\
\hline Columba cayennensis - B & 210 & 150 & 91 & 24 \\
\hline Columba picazuro - A & 98 & 327 & 533 & 416 \\
\hline Columbina squammata $-\mathrm{B}+$ & - & - & 8 & 16 \\
\hline Columbina talpacoti - B & 164 & 133 & 290 & 91 \\
\hline Claravis pretiosa $-\mathrm{T}$ & 43 & 41 & 6 & - \\
\hline Leptotila verreauxi & 153 & 162 & 188 & 60 \\
\hline Leptotila rufaxilla & 4 & - & - & - \\
\hline Zenaida auriculata - A & - & 3 & 2 & 3 \\
\hline Piaya cayana & 89 & 51 & 58 & 50 \\
\hline Tapera naevia - B & 59 & 15 & 25 & 8 \\
\hline Dromococcyx pavoninus & 1 & - & - & - \\
\hline Guira guira - A & 289 & 629 & 414 & 202 \\
\hline Crotophaga ani-B & 152 & 187 & 219 & 98 \\
\hline Coccyzus euleri - T & - & - & 1 & - \\
\hline Coccyzus melacoryphus - BT & 5 & - & 1 & - \\
\hline Aratinga leucophthalmus $-\mathrm{S}$ & 43 & 24 & 65 & 101 \\
\hline Forpus xanthopterygius - B & 251 & 355 & 258 & 112 \\
\hline Pionus maximiliani & 159 & 350 & 461 & 288 \\
\hline Amazona aestiva $-\mathrm{V}$ & 1 & - & - & - \\
\hline Strix hylophila -+ & 3 & - & 14 & - \\
\hline Pseudoscops clamator -+ & - & - & 3 & 1 \\
\hline Otus choliba - B & 3 & 6 & 6 & 2 \\
\hline
\end{tabular}


TABLE 1 (Continued)

\begin{tabular}{|c|c|c|c|c|}
\hline Species & 1 & 2 & 3 & 4 \\
\hline Nyctibius griséus $-\mathrm{BT}+$ & 1 & 4 & 8 & - \\
\hline Lurocalis nattereri $-\mathrm{T}$ & 16 & 26 & 23 & 9 \\
\hline Nyctidromus albicollis - B & 10 & 46 & 198 & 59 \\
\hline Caprimulgus parvulus - AT & 3 & 6 & - & - \\
\hline Hydropsalis torquata - A & 12 & 37 & 112 & 30 \\
\hline Chaetura meridionalis - AT & 14 & 29 & 7 & - \\
\hline Tachornis squamata - AV+ & - & - & - & 1 \\
\hline Phaethornis pretrei & 52 & 77 & 82 & 58 \\
\hline Colibri serrirostris - B & - & - & 1 & - \\
\hline Melanotrochilus fuscus & 44 & 56 & 61 & 9 \\
\hline Anthracothorax nigricollis $-\mathrm{T}$ & 11 & 13 & 2 & 5 \\
\hline Chlorostilbon aureoventris - B & 11 & 18 & 26 & 8 \\
\hline Aphantochroa cirrochloris & 73 & 80 & 117 & 26 \\
\hline Eupetomena macroura - B & 5 & 7 & 2 & - \\
\hline Amazilia lactea & 280 & 174 & 90 & 28 \\
\hline Amazilia versicolor & 59 & 60 & 67 & 3 \\
\hline Thalurania glaucopis - S & 4 & 6 & 3 & - \\
\hline Heliomaster squamosus & 1 & - & 4 & 4 \\
\hline Leucochloris albicollis $-\mathrm{S}$ & 10 & 2 & 4 & - \\
\hline Calliphlox amethystina & 12 & 14 & 13 & 4 \\
\hline Ceryle torquata $-\mathrm{C}$ & 29 & 22 & 21 & 7 \\
\hline Chloroceryle amazona - C & 19 & 33 & 39 & 14 \\
\hline Chloroceryle americana $-\mathrm{C}$ & 28 & 37 & 32 & 7 \\
\hline Bucco chacuru - A & 6 & - & - & - \\
\hline Ramphastos toco & - & - & - & 8 \\
\hline Picumnus albosquamatus & 64 & 30 & 56 & 9 \\
\hline Colaptes campestris - A & 39 & 67 & 72 & 70 \\
\hline Colaptes melanochloros $-\mathrm{B}$ & 18 & 47 & 49 & 23 \\
\hline Melanerpes candidus - B & 53 & 58 & 96 & 69 \\
\hline Veniliornis passerinus & 94 & 80 & 102 & 47 \\
\hline Dryocopus lineatus & 52 & 34 & 46 & 29 \\
\hline Campephilus robustus & 17 & 8 & 8 & 4 \\
\hline Sittasomus griseicapillus & 85 & 87 & 97 & 46 \\
\hline Dendrocolaptes platyrostris & 30 & 34 & 19 & 5 \\
\hline Campylorhamphus falcularius $-\mathrm{V}+$ & - & 1 & - & - \\
\hline Furnarius rufus - A & - & 9 & - & 25 \\
\hline Synallaxis spixi- B & 144 & 123 & 92 & 54 \\
\hline Synallaxis frontalis - B & 154 & 165 & 139 & 82 \\
\hline Synallaxis ruficapilla & 49 & 69 & 79 & 41 \\
\hline Certhiaxis cinnamomea $-\mathrm{C}$ & 137 & 151 & 140 & 67 \\
\hline
\end{tabular}


TABLE 1 (Continued)

\begin{tabular}{|c|c|c|c|c|}
\hline Species & 1 & 2 & 3 & 4 \\
\hline Automolus leucophthalmus & 56 & 43 & 19 & 3 \\
\hline Phacellodomus ferrugineigula $-\mathrm{C}$ & 79 & 52 & 43 & 34 \\
\hline Lochmias nematura & 19 & 12 & 1 & 4 \\
\hline Xenops rutilans & 36 & 52 & 58 & 6 \\
\hline Mackenziaena severa & 75 & 55 & 53 & 40 \\
\hline Taraba major - B & - & - & - & 7 \\
\hline Thamnophilus doliatus - B & 6 & 14 & 29 & 42 \\
\hline Thamnophilus caerulescens & 237 & 203 & 185 & 73 \\
\hline Thamnophilus ruficapillus - A & - & 2 & 3 & - \\
\hline Dysithamnus mentalis & 2 & 5 & 13 & 3 \\
\hline Pyriglena leucoptera & 46 & 25 & 30 & 8 \\
\hline Conopophaga lineata & 83 & 65 & 84 & 39 \\
\hline Antilophia galeata & 19 & - & 2 & - \\
\hline Chiroxiphia caudata & 12 & 10 & 63 & 7 \\
\hline Schiffornis virescens & 2 & - & - & - \\
\hline Tityra cayana - $\mathrm{T}$ & 17 & 26 & 14 & 2 \\
\hline Tityra inquisitor $-\mathrm{S}$ & 1 & 3 & 9 & - \\
\hline Pachyramphus validus - B & 85 & 36 & 17 & 8 \\
\hline Pachyramphus viridis - BS & 5 & 5 & - & - \\
\hline Pachyramphus polychopterus - BT & 106 & 102 & 68 & 37 \\
\hline Phyllomyias virescens $-\mathrm{S}+$ & 4 & 8 & 1 & 1 \\
\hline Phyllomyias fasciatus - BV & - & 4 & - & - \\
\hline Myiopagis viridicata $-\mathrm{T}$ & 136 & 115 & 67 & 49 \\
\hline Myiopagis caniceps & 182 & 208 & 145 & 61 \\
\hline Elaenia flavogaster - B & 94 & 88 & 90 & 50 \\
\hline Elaenia spectabilis - CT & 17 & 1 & 1 & - \\
\hline Elaenia obscura - BS & 6 & 20 & 9 & - \\
\hline Elaenia mesoleuca - s & 21 & 12 & - & 3 \\
\hline Elaenia chiriquensis - BV & 2 & - & - & - \\
\hline Elaenia parvirostris $-\mathrm{s}$ & 3 & 1 & - & - \\
\hline Serpophaga subcristata - B & 43 & 11 & - & 4 \\
\hline Camptostoma obsoletum & 107 & 92 & 88 & 44 \\
\hline Todirostrum cinereum - B & 1 & 1 & 24 & 9 \\
\hline Todirostrum poliocephalum & 73 & 37 & 52 & 32 \\
\hline Capsiempis flaveola & 55 & 44 & 63 & 17 \\
\hline Hemitriccus nidipendulus - B & 5 & - & - & - \\
\hline Myiornis auricularis & 31 & 32 & 13 & 4 \\
\hline Myiophobus fasciatus - B & 75 & 44 & 67 & 32 \\
\hline
\end{tabular}


WILLIS, E. O.

TABLE 1 (Continued)

\begin{tabular}{|c|c|c|c|c|}
\hline Species & 1 & 2 & 3 & 4 \\
\hline Tolmomyias sulphurescens & 166 & 130 & 135 & 62 \\
\hline Corythopis delalandi & 4 & 1 & 2 & - \\
\hline Knipolegus cyanirostris - BS & 8 & 2 & 3 & 1 \\
\hline Fluvicola leucocephala-C & 96 & 87 & 90 & 50 \\
\hline Fluvicola nengeta - C+ & - & 7 & 52 & 73 \\
\hline Pyrocephalus rubinus - AS & - & - & 1 & - \\
\hline Colonia colonus & 79 & 39 & 59 & 41 \\
\hline Gubernetes yetapa - C & 1 & - & - & - \\
\hline Hirundinea ferruginea - A+ & - & - & - & 1 \\
\hline Contopus cinereus & 77 & 93 & 58 & 26 \\
\hline Lathrotriccus euleri & 150 & 164 & 117 & 53 \\
\hline Cnemotriccus bimaculatus - B & - & 1 & - & - \\
\hline Platyrinchus mystaceus & 5 & 11 & 14 & 3 \\
\hline Leptopogon amaurocephalus & 60 & 61 & 41 & 17 \\
\hline Myiarchus ferox - B & 34 & 20 & 42 & 26 \\
\hline Myiarchus tyrannulus - B+ & 14 & 14 & 37 & 25 \\
\hline Myiarchus swainsoni - T & 76 & 73 & 44 & 20 \\
\hline Sirystes sibilator -+ & 2 & 1 & - & - \\
\hline Satrapa icterophrys - B & 61 & 21 & 13 & 6 \\
\hline Machetornis rixosus - A & 46 & 110 & 74 & 40 \\
\hline Pitangus sulphuratus - B & 284 & 324 & 305 & 138 \\
\hline Myiozetetes similis - C & 118 & 136 & 135 & 71 \\
\hline Megarynchus pitangua - B & 106 & 145 & 134 & 66 \\
\hline Tyrannus melancholicus - BT & 145 & 147 & 132 & 98 \\
\hline Tyrannus albogularis - ATV+ & - & 1 & 1 & - \\
\hline Tyrannus savana - BT & 9 & 8 & 10 & 2 \\
\hline Myiodynastes maculatus - BT & 156 & 154 & 129 & 63 \\
\hline Empidonomus varius - BT & 90 & 95 & 69 & 33 \\
\hline Legatus leucophaius - TV+ & - & 4 & - & - \\
\hline Tachycineta leucorrhoa - C & 42 & 29 & 22 & 9 \\
\hline Tachycineta albiventer $-\mathrm{C}+$ & - & - & 56 & 37 \\
\hline Progne chalybea - As & 8 & - & - & 246 \\
\hline Progne subis - AN & 163 & 32 & 41 & 3 \\
\hline Progne tapera - AT & 34 & 54 & 29 & 32 \\
\hline Notiochelidon cyanoleuca - A & 827 & 1652 & 1362 & 732 \\
\hline Stelgidopteryx ruficollis - B & 40 & 33 & 37 & 41 \\
\hline Hirundo rustica - AN & 2 & - & - & - \\
\hline Cyanocorax chrysops -+ & 3 & - & - & - \\
\hline Cyanocorax cristatellus - B & 2 & 5 & 45 & 104 \\
\hline
\end{tabular}


TABLE 1 (Continued)

\begin{tabular}{|c|c|c|c|c|}
\hline Species & 1 & 2 & 3 & 4 \\
\hline Troglodytes aedon - B & 309 & 338 & 354 & 172 \\
\hline Donacobius atricapillus - C & 108 & 105 & 93 & 17 \\
\hline Mimus saturninus - A & - & - & - & 16 \\
\hline Catharus fuscescens $-\mathrm{N}+$ & 1 & - & - & - \\
\hline Platycichla flavipes $-\mathrm{B}+$ & 2 & 33 & 2 & 3 \\
\hline Turdus rufiventris $-\mathrm{B}$ & 60 & 77 & 32 & 10 \\
\hline Turdus leucomelas - B & 204 & 162 & 246 & 92 \\
\hline Turdus amaurochalinus - B & 125 & 162 & 75 & 25 \\
\hline Turdus albicollis & 22 & 34 & 7 & - \\
\hline Turdus nigriceps $-\mathrm{s}$ & 7 & 37 & 7 & 1 \\
\hline Cyclarhis gujanensis & 543 & 446 & 346 & 135 \\
\hline Vireo olivaceus $-\mathrm{T}$ & 205 & 133 & 55 & 19 \\
\hline Hylophilus amaurocephalus - B & 12 & 5 & - & 1 \\
\hline Passer domesticus - A & 11 & 22 & 51 & 92 \\
\hline Spinus magellanicus $-\mathrm{S}+$ & 5 & 3 & 3 & - \\
\hline Zonotrichia capensis - B & 252 & 164 & 273 & 121 \\
\hline Ammodramus humeralis - A & - & - & 6 & 5 \\
\hline Emberizoides herbicola - A & 2 & - & - & 1 \\
\hline Arremon flavirostris & 1 & 1 & 4 & 9 \\
\hline Coryphospingus cucullatus & 15 & 3 & 2 & 16 \\
\hline Tiaris fuliginosa & 9 & 6 & 5 & 6 \\
\hline Sporophila caerulescens - B & 30 & 12 & 133 & 70 \\
\hline Sporophila lineola - BT & - & - & 1 & 1 \\
\hline Volatinia jacarina - B & 4 & 5 & 9 & 23 \\
\hline Saltator similis & 75 & 54 & 43 & 15 \\
\hline Passerina glaucocaerulea - BS & 1 & 1 & - & - \\
\hline Schistochlamys ruficapillus - A & 2 & - & 8 & - \\
\hline Thlypopsis sordida & 65 & 69 & 49 & 16 \\
\hline Pyrrhocoma ruficeps $-\mathrm{S}$ & - & 1 & - & - \\
\hline Euphonia chlorotica & 73 & 94 & 109 & 42 \\
\hline Euphonia musica - S & - & - & 44 & 2 \\
\hline Euphonia violacea $-\mathrm{S}$ & 6 & 12 & 26 & 3 \\
\hline Tangara peruviana $-\mathrm{s}+$ & - & 1 & 1 & - \\
\hline Tangara cayana $-\mathrm{B}$ & 144 & 172 & 177 & 45 \\
\hline Pipraeidea melanonota $-\mathrm{S}$ & 48 & 45 & 15 & 1 \\
\hline Nemosia pileata & 121 & 140 & 85 & 10 \\
\hline Thraupis sayaca - B & 314 & 553 & 484 & 155 \\
\hline Thraupis palmarum - $\mathrm{B}+$ & 3 & 27 & 25 & 12 \\
\hline
\end{tabular}


TABLE 1 (Continued)

\begin{tabular}{|c|c|c|c|c|}
\hline Species & 1 & 2 & 3 & 4 \\
\hline Ramphocelus carbo - B & 70 & 87 & 133 & 59 \\
\hline Piranga flava -+ & 48 & 31 & 19 & 22 \\
\hline Habia rubica & 61 & 88 & 48 & 6 \\
\hline Tachyphonus coronatus & 56 & 54 & 118 & 59 \\
\hline Trichothraupis melanops & 103 & 94 & 62 & 30 \\
\hline Hemithraupis guira - V+ & 4 & 4 & - & - \\
\hline Hemithraupis ruficapilla & 155 & 168 & 142 & 33 \\
\hline Tersina viridis $-\mathrm{Bs}+$ & 1 & 4 & 27 & 2 \\
\hline Dacnis cayana & 44 & 60 & 86 & 8 \\
\hline Dacnis nigripes $-\mathrm{s} \mathrm{V}+$ & - & - & 3 & - \\
\hline Conirostrum speciosum & 258 & 266 & 195 & 40 \\
\hline Cyanerpes cyaneus $-\mathrm{s}+$ & 2 & 5 & 3 & - \\
\hline Coereba flaveola - B & 212 & 186 & 165 & 64 \\
\hline Geothlypis aequinoctialis - C & 75 & 45 & 89 & 63 \\
\hline Basileuterus flaveolus & 175 & 228 & 208 & 88 \\
\hline Basileuterus hypoleucus & 279 & 214 & 178 & 68 \\
\hline Basileuterus culicivorus $-\mathrm{s}$ & 3 & - & - & - \\
\hline Parula pitiayumi & 127 & 80 & 78 & 37 \\
\hline Molothrus bonariensis - A & 34 & 34 & 33 & 28 \\
\hline Agelaius cyanopus - C & 22 & 3 & 3 & - \\
\hline Agelaius ruficapillus $-\mathrm{C}$ & 7 & 5 & 50 & 117 \\
\hline Icterus cayanensis - B+ & - & - & 2 & 18 \\
\hline Cacicus haemorrhous & 2 & - & - & - \\
\hline DAYS & 61 & 63 & 57 & 28 \\
\hline DAY HOURS & 294.7 & 313 & 270.9 & 144.8 \\
\hline NIGHT HOURS & $2+$ & $6+$ & $19+$ & $8+$ \\
\hline SPECIES & 223 & 210 & 219 & 205 \\
\hline
\end{tabular}

1 - March 1982-Feb. 1986.

2 - March 1986-Feb. 1990.

3 - March 1990-Feb. 1996.

4 - March 1996-Feb. 2001. 


\section{"Vagrants"}

Eleven Horto species are considered vagrants: Platalea on the pond on 12/4/92; Spizaetus singing atop the eucalyptus on 14/8/83; Amazona flying over on 5/5/85; Campylorhamphus singing in understory by the N bridge on 6/10/87; Phyllomyias fasciatus at fruit of Alchornea glandulosa from 14/ 9-2/10/86; Tyrannus albogularis at these fruits 28/ 9/86 and 14/11/93; Legatus, too, on 28/9-12/10/ 86 , as were Hemithraupis guira $2 / 10$ to $26 / 10 / 86$ (also seen, however, $8 / 8$ and $18 / 8 / 82$, high in the eucalyptus near $\mathrm{N}$ bridge) and Dacnis nigripes (2/ 10 to 9/10/94; even by Antunes, 2000, at eucalyptus flowers 3/10/98). Elaenia chiriquensis (15/9 and 29/10/82) may have been a wanderer too, but is a migrant like several of the other species considered vagrants. Tachornis checking Roystonea regia royal palms at the lake on 14/10/97 is the southernmost record for the species, which is common mostly near palm groves on the north of the state.

T. albogularis and E. chiriquensis do breed regularly just northwest, near cerrados of Itirapina, where a few Amazona pairs occur. Hemithraupis guira hybridizes with $H$. ruficapilla off north, $D$. nigripes and Legatus breed off to the south and winter off to the north, while Spizaetus, Campylorhamphus, and Phyllomyias fasciatus occur near the coast but are rare inland, due to deforestation. Cyanerpes would be called a vagrant, were it not that a pair were on the dam in eucalyptus flowers on 18/8/85, 27/7/86 (Willis, 1987), and $16 / 8 / 87$, while a single female was there on $31 /$ $7 / 88$ (Willis \& Oniki, 1993) and in nearby Alchornea on 2/10-9/10/94.

A similar number of "vagrants" (10) was recorded for Fazenda S. José, including some $E$. chiriquensis, one P. fasciatus, and one Amazona, plus 3 other Psittacidae wandering south or north, a coastal antshrike, a cerrado small flycatcher, a grosbeak much captured as a pet, and a similarly captured summer Sporophila lineola (nonvagrant in the Horto). For both areas, there are 17 vagrants, so only 290 species are or were more or less regular in the area just east of Rio Claro.

Very limited numbers are recorded for certain southern forest species that occasionally visit in winter (Euphonia musica, E. violacea, Pyrrhocoma in both areas, plus Tangara peruviana from the Horto) or while migrating (Elaenia parvirostris, as for southern open-zone species, Pyrocephalus plus Donacospiza, at S. José). A dry-forest nightjar and open-area one for S. José, plus a snipe, heron, and kite of open zones there, were also rarely recorded. In the Horto, Botaurus was rare, as were Amaurolimnas, migrant Tringa and Catharus from North America, plus Gampsonyx near the campus. However, these species may be regular, as they occur elsewhere in central São Paulo and are not considered vagrants.

\section{Absences 1982-2001}

From March 1982 to 1986,15 of the 165 forest and border species were not recorded, leaving 150 "early" species (Table 1, column 1). To Feb. 1990 (Column 2), 22 went unrecorded, leaving 143 species; to Feb. 1996, 21 species (144) and to Feb. 2001, 36 also were not recorded (129 species recorded). Similar decreases over the years were recorded in São José and in a Campinas woodlot (Willis, 1979, vs. Aleixo \& Vielliard, 1995).

Only 3 forest and border species in 198286 were probably really absent, invading later: Ramphastos (first near on lake on 11/10/98), Taraba (first at E scrub on 7/7/98), and Icterus (first on 3/6 and 24/6/90, regular from 10/97 on near lake). The first two have also invaded São José in recent years (Willis \& Oniki, 2002a). The other "missing" species include Scardafella and Sporophila lineola present around town, but perhaps slow to invade close to the lake (former noted mostly from 1990 on, latter only in 1994 and 2001, but subject to caging), and a number of migrants rarely recorded in any one or few years.

Four open-area birds from near the Horto moved in near the lake recently, also: Zenaida is common in and around town, but so far only flies over the eucalyptus or open lake region; Furnarius was briefly present there only in 1987 and 1989, nesting from $21 / 11 / 99$ on, though common in town and present near São José much earlier; one Hirundinea found the deserted church and other tall buildings only on 4/2/01 (species more common in distant dry cliff zones northwest of Rio Claro); Mimus is common around town but entered the lake area from 24/11/97 on. Two Falco only occur over the campus or cane, not at the open lake area, but were present from 1982 on outside. From 198690 on, there were no more Leptotila rufaxilla (last 30/9/82), Dromococcyx (22/8/82), Schiffornis (30/ 
9/82), Hemitriccus (3/10/82), Catharus (23/2/84), and Cyanocorax chrysops (15/9/82 and 27/10/85). Several were located more in swamp woods along the west and north edges (areas checked less after 1982) and may still be present. Cacicus (3/10 and 29/10/82) vanished, as happened in Fazenda São José. Rare winter Basileuterus culicivorus (15/7/ $82,8 / 6$ and 18/7/84) were recorded only one winter after the unusually wet "El Niño" of 1982-83.

Some water birds disappeared: Botaurus (only on 16/9 and 3/11/84), Amaurolimnas (15/ $3 / 84$; it may still occur in the swamp woods), and Tringa melanoleuca (only on 6/3/82). Bucco (last recorded on 27/10/85) of open zones around the Horto seemed to disappear, as occurred near São José (Willis \& Oniki, 2002a); Gubernetes (last recorded on 3/10/82) still occurs in wet swales in cane near São José, but in low numbers; Hirundo (only on 13/10/85) may be a similar case, as is Emberizoides (only on 30/9/82 and 23/3/97).

In 1990-96, Buteo brachyurus (last 5/7/87) seemed gone, as likewise near São José, but still flies over town at times. Pachyramphus viridis (last seen on $16 / 7 / 89 ; 14 / 4$ to $18 / 9$ in earlier years, perhaps wintering from edges off to the south) seemingly disappeared, as was the case with Elaenia parvirostris (migrant from south; seen on 14/4/84-17/5/87 and 27/10/85; still registered in São José), Cnemotriccus (only on 6/10/87, edge; still seen in São José edges), Sirystes (only on 8/ 8 and $18 / 8 / 82$, and $16 / 7 / 89$, treetop flocks at the north creek, maybe a rare winter bird or looking for a habitat), Passerina (only on 8/8/82 and 14/ 6/86, a rare winter bird regionally; also disappeared from S. José), and Pyrrhocoma (only on 18/9/88, another rare winter bird, also disappeared from S. José). Agelaius cyanopus vanished with cat-tail clearing around the lake (last seen on 4/11/93, and rare from 5/7/87 on; Antunes, 1996, registered the bird until 1/94) while Porphyrula vanished for the same reason, from 1988 on with one seen on 10/ $11 / 95$ and a few in 1999-2000 (Ferrari, 1985, showed it seeks shade during hot midday hours on the lake), while Aramus got much less common.

By 1996-2001, Crypturellus tataupa seemed to disappear (last seen on 17/9/95, perhaps because of vagrant dogs or drought) as did Cathartes (seen up to 2/20/94; had bred in 1982 but always uncommon in the region, perhaps killed by occasional hunters shooting Coragyps), Gampsonyx (up to 20/ $7 / 92$ and later near the campus), Claravis (last seen on 11/1/94; earlier on 30/7-13/4, in summer, nested; last sighted also in S. José after 1994 seeding of bamboos), Strix (up to 11/1/94; block cut from its territory at SE corner in 1991-92), Thalurania (AprilSept. earlier), Leucochloris (rare from 15/3-18/8, last seen on 3/7/94; Antunes, 2000, registered one on 23/ $6 / 98$, however), Elaenia obscura (last seen on 10/ $11 / 95$, winter or from 17/5-30/9 were the only previous records), Corythopis (always rare, seen up to 1993 , but recorded at another site in 2000 by a student), Turdus albicollis (last seen on 15/5/94, always uncommon though less so in March-Oct.; also vanished from S. José), and Spinus (15/4-29/10).

Water birds that disappeared were Pilherodius (last seen on 17/9/95 and up to $12 / 95$ by Antunes, 1996), Elaenia spectabilis (last seen on 17/9/95; rare creekside summer bird, 15/9/82-12/ 84; also disappeared from São José). The perhaps unusually wet 1982 depressions have gradually dried out, despite El Niño rains in 1987, 1992, and 1997, possibly explaining these cases.

Some of these species have been recorded again in small numbers in 2001-02, with El Niño rains: Cathartes, Pilherodius, Dromococcyx, Buteo brachyurus, Agelaius cyanopus (marshes off east), Crypturellus tataupa, Elaenia obscura, Turdus albicollis, and Antilophia.

\section{Numerical analysis}

For ducks, feeding Cairina and introducing a few white tame birds at the lake in 1993-94 caused considerable growth in numbers (Table 1), which increased 10-20 per visit, the females appearing with young and some hybrids. Dendrocygna viduata flocks mostly rest on sandbars, flying out at night to feed; they are thus more common in the dry winter and in dry La Niña years, as shown by the 250 on $7 / 7 /$ 98 and 150 on 28/5/00, and even 291 on 9/9/95 (Antunes, 1996). Large flocks rest by day on a protected island of Lago Azul in central Rio Claro, as an alternative. D. autumnalis has been reported eastward in the State recently (L. O. MarcondesMachado, pers. comm.), and finally appeared on 4/ 2/01. Amazonetta and spring/summer Nomonyx occur in small numbers, the latter rare in 1989-92 and 199900 with water-lily cleanup; both seem less common whenever aggressive Cairina are present. 
Fish-eating waterbirds are rare, with one or another anhinga or cormorant in recent years; the latter fish more in deep-water Lago Azul. Podilymbus singles or pairs (April to Oct., summers of 1985-86 and 1991-95, too) attack occasional Tachybaptus, present mostly June-Sept. (absent in 1987-88 and 1990-94). Scattered egrets wander at the edges, with flocks only in Oct. 1994 at drying polluted pools downcreek from the lake, due to closing its gate to raise water levels. Butorides fish atop water lilies, once even throwing a Salvinia leaf in to attract fish, and disappear in months after the lilies are cleared off as well as some dry winter months (1989-91 following big clearing of cattails in mid-1988 on; less common from 1995 on). Tigrisoma and Pilherodius upcreek seemed to disappear after the cat-tail cleanup, with egrets in the open taking their place; but the first two had been rather uncommon even in the early 1980s. Kingfishers visited in small numbers, but rarely even 2 birds of a species.

Snail-eating Rostrhamus (first 26/10/86) were present mainly after water-lily cleanups 1988 on, but disappeared with a similar cleanup in the 2000 drought year; when two birds appeared, each of them took half the lake. Aramus have been uncommon after the water-lily and cat-tail (for midday shade and nesting) loss in 1988, and began to disappear in dry winters.

Creek and swamp Mesembrinibis increased from 26/11/89 on after cat-tail borders were cleared, but Laterallus among the cat-tails vanished (except at other small marshes); Aramides spp. and Rallus became uncommon (rare especially during the 2000 La Niña drought), and Porphyrula and Agelaius cyanopus vanished (see above). Jacana was still common in spite of occasional water-lily removal, even feeding up on the mowed lawns, but the 1999 lily removal left only one lawn-edge pair for a year. Gallinula was even more common with cat-tail removal, despite occasional lily removals, and also used mowed lawns early in the morning before tourists arrived. Cat-tail removal allowed Tringa solitaria to visit in Aug.-Nov. and March during migration, and even remain all winter in 1991-92; T. flavipes visited on 24/2/91. Certhiaxis survived cat-tail removal by moving to nearby grass or bamboo clumps. Phacellodomus and Geothlypis mostly vanished but stayed at other small marshes (the latter uses uncut sugar cane). Pilherodius and
Elaenia spectabilis just disappeared (above) as happened in S. José. Donacobius of marshes at the lake survived despite several cat-tail removals, but dropped off recently (last seen on 11/1/99 before the La Niña drought). Arundinicola stayed, and Fluvicola (a bird that has come into humid SE Brazil recently with cutting or "desertification" around water areas, see Willis, 1991) entered from 9/4/89 on. Agelaius ruficapillus is now using regrown cat-tails, because of or despite $A$. cyanopus loss with cat-tail removal; the first invaded the state with deforestation, for it flies to distant or nearby pastures for food and uses cat-tails only to nest.

Around the lake, Myiozetetes continues common. Tachycineta leucorrhoa dropped off (it mostly visited from $13 / 4$ to $3 / 12$, finding no nest holes except by similar ponds with bamboo near S. José; a Colaptes melanochloros at the lake threw out swallow material $14 \mathrm{~m}$ up, on 20/8/93) even before $T$. albiventer arrived (26/3/90). The latter needs a dead tree, floating plastic bottle, or shack in the water to sit between foraging sessions, and vanishes with cleanup of perches, hence being affected by human activity. Other passing swallows, which use the lake to drink and bathe at mid-day, continue common (Notiochelidon nests and roosts in abandoned buildings) or uncommon (Stelgidopteryx, with few gullies to nest in and hence often rare in summer; Progne tapera, which has no ovenbird nests to steal, but flies over in March-April, at times from 30 Sept. on, once beginning on 10/7/84; $P$. chalybea, without nests except in the city nearby, occuring in March-April in flocks, and at times from Sept. on). $P$. subis once roosted in huge flocks in Oct.-May in trees of a city plaza, but may have been run off in 1994 because of its feces (recent records for the lake only at 4/11/96 and 4/11/99). Other northern swallows are occasional over cane fields in the region.

Notable is that several open-area species, including ones common around campus and town, have found the interior open areas near the lake only in recent years: Mimus, Furnarius, Pyrocephalus, Hirundinea. Border Sporophila lineola, Icterus, Cyanocorax cristatellus, Todirostrum cinereum, and Scardafella also moved in recently. Both Chaetura and Zenaida of the city, like the Progne, appear over the lake area but do not stay. Athene, common on campus next to the Horto, has not yet been recorded in the Horto. Several species of 
surrounding cane areas (or grassy or swampy open zones) have also not moved into the lake open region: Crypturellus parvirostris (but it moves to tall grass under young eucalyptus when cows are removed), Nothura, Bubulcus, Cariama, 2 Falco, Hydropsalis, Bucco, Thamnophilus ruficapillus, Schistochlamys, Ammodramus, and Emberizoides. Neither have border Gampsonyx moved in. Several other open-zone species have been regular in the central semiopen lake area: Syrigma, Vanellus, Milvago, Columba picazuro (increasing in São Paulo with desert-like open zones, see Willis \& Oniki, 1987), Guira, Colaptes campestris, Machetornis, Passer, and Molothrus.

Resident border species that have always reached the central open zone are 31: Coragyps, Accipiter, 2 Buteo, Caracara, Herpetotheres, Columba cayennensis, Columbina, Crotophaga, Forpus, Otus, Nyctidromus (decreasing in recent years near the $\mathrm{W}$ entrance by the city, likely victim of city cats, see Ingels et al., 1999), Colibri, Eupetomena, Chlorostilbon, Colaptes melanochloros, Melanerpes, Synallaxis spixi, Pachyramphus validus, Elaenia flavogaster, Serpophaga (common only during wet 1982-84), Myiophobus, Myiarchus ferox and M. tyrannulus, Stelgidopteryx (see above), Troglodytes (common even in tall eucalyptus forest), Turdus rufiventris, T. leucomelas (a dry-region species that is increasing, and perhaps causing decreases noted in other thrushes; see Bencke \& Grillo (1995) for increases southward and Willis \& Oniki (2002b) for increases in Espírito Santo), T. amaurochalinus, and Zonotrichia. Summer species of borders are 4: Ictinia (14/921/1), Nyctibius (26/9-13/2), Pachyramphus polychopterus (during 28/8-18/6 with records for 4/7/82, 5/7/87, 31/7/88), Myiodynastes (29/8-23/ 4), and Empidonomus (14/9-24/3). Winter species are Elaenia obscura (see above), Knipolegus (7/ 5-30/9), Platycichla (3/6-27/10 except during mass fruiting of the palm Archontophoenix cunninghamia planted by the lake, in 1987, when one nest succeeded but a 1988 one failed, present from 30/ 8/87 to 15/11/88; Turdus leucomelas dominated a similar fruiting in 2000 , so there were no Platycichla; 2000 was a very dry La Niña winter).

Border species that seem not to reach the interior open lake zone are 11: Tapera, Synallaxis frontalis, Thamnophilus doliatus (though common even in town, see Oniki et al., 2002), Hemitriccus, Cnemotriccus, Hylophilus, Coryphospingus, Sporophila caerulescens, and Volatinia (weed removal must explain lack of the last 3 species), plus summer Coccyzus melacoryphus (29/10-27/2) and winter Pachyramphus viridis (above).

As noted above, 9 of 68 border species have not been recorded recently (Gampsonyx, Buteo brachyurus, Coccyzus melacoryphus, Nyctibius, Eupetomena, Pachyramphus viridis, Elaenia obscura, Hemitriccus, Passerina), though Eupetomena is common in the city and Antunes 2000 records it at eucalyptus flowers, so it must still occur. Thirteen species have decreased in recent years: Columba cayennensis, Tapera, Pachyramphus validus and $P$. polychopterus, Serpophaga, Knipolegus, Satrapa, Myiodynastes, Empidonomus, Turdus rufiventris and T. amaurochalinus, Hylophilus, and Coereba.

Slight or no decreases were noted for 25 border species: Coragyps, Ictinia, Accipiter, Buteo magnirostris, Herpetotheres, Forpus, Otus, Nyctidromus, Chlorostilbon, Synallaxis spixi and S. frontalis, Elaenia flavogaster, Myiophobus, Pitangus, Megarynchus, Tyrannus melancholicus and T. savana, Stelgidopteryx, Troglodytes, Platycichla, Zonotrichia, Coryphospingus, Tangara cayana, Thraupis sayaca, and Tersina. Fifteen species increased: Caracara, Columbina, Crotophaga, Colaptes melanochloros, Melanerpes, Thamnophilus doliatus, Todirostrum cinereum, Myiarchus ferox and M. tyrannulus, Cyanocorax cristatellus, Turdus leucomelas, Sporophila caerulescens, Volatinia, Thraupis palmarum, and Ramphocelus. "New" border species are Scardafella, Colibri and Cnemotriccus (once each), and Sporophila lineola and Icterus (above).

Only 97 species in Table 1 are "forest" species rather than vagrants or water, open, or border birds. Some 8 of the 97 are mere passing migrants: Chondrohierax (10/11/95, 21/10, and 9/12/00), Harpagus (18/8/91, 14/10-8/11, 28/3), Tityra inquisitor (18/8-14/11, plus $31 / 7 / 88$ and $18 /$ 6/89), Elaenia mesoleuca (27/9-10/11, 26/4), E. parvirostris (27/10, 14/4-17/5), Turdus nigriceps (30/8-2/11 and 12/4-24/6), Tangara peruviana (16/ 8/87 and 3/6/90), Cyanerpes (see above), and perhaps Dacnis nigripes (called "vagrant" above). Some 10 others are winter visitors from the south or nearby: Aratinga (March-Sept., with occasional records for other months recently and which is 
increasing in the region, where it occurs all year in cerrado border zones), Thalurania (above; nests in S. José), Leucochloris (above), Phyllomyias virescens (rare 14/4-22/8), Spinus (above), Pyrrhocoma (above), Euphonia musica (24/3-13/ 8), E. violacea (16/6-7/10), Pipraeidea (13/4-18/ 9, mostly in 1982 and 1986), and Basileuterus culicivorus (above). One species is a migrant from North America (Catharus, above).

Subtracting the above 19 migrants, only 78 forest species probably breed in the Horto. Of these, 8 are summer species: Claravis (above), Coccyzus euleri (above), Lurocalis (26/9-24/3), Anthracothorax (6/10-12/4, less common recently), Tityra cayana (9/10-14/4, also $1 / 6$ and 14/6/86 and 16/8/87; uncommon recently), Myiopagis viridicata (15/9-7/7, staying to early winter in some years), Myiarchus swainsoni (30/8-1/3), and Vireo (29/ $8-14 / 4$, less common recently). Thus, 70 species are permanent forest residents.

Of these 70 species, 12 have disappeared in recent years (Crypturellus tataupa, Cathartes, Leptotila rufaxilla, Dromococcyx, Strix, Antilophia, Schiffornis, Corythopis, Sirystes, Cyanocorax chrysops, Turdus albicollis, Cacicus), as noted above. Decreased numbers have been registered recently for 29 species: Leptodon, Pseudoscops, Melanotrochilus, 2 Amazilia, Campephilus, Dendrocolaptes, Automolus, Lochmias, Xenops, Pyriglena, Chiroxiphia, Myiopagis caniceps, Todirostrum poliocephalum, Myiornis, Contopus (nests in huge old eucalyptus, and rarely in native forests in the region), Leptopogon, Cyclarhis (common in tall eucalyptus, more so than in native canopy), Saltator, Thlypopsis, Nemosia, Piranga (one of the few species that really prefers eucalyptus crowns to normal treetops, being a cerrado bush specialist originally), Habia, Trichothraupis, Hemithraupis ruficapilla, Dacnis cayana, Conirostrum, Basileuterus hypoleucus, and Parula. Canopy mixed flocks of M. caniceps, Nemosia, H. ruficapilla, and Conirostrum wander in tall eucalyptus early, descending to the native tall understory at midday; understory Habia, Automolus, Sittasomus, and B. hypoleucus flocks wander all day. A likely major factor in decreases was the cutting of large areas of eucalyptus in the late 1980s and early 1990s.

Slight or no decreases were noted for 24 species: Micrastur (rare), Leptotila verreauxi,
Piaya, Aphantochroa, Heliomaster, Calliphlox, Picumnus, Veniliornis, Dryocopus, Sittasomus, Synallaxis ruficapilla, Mackenziaena, Thamnophilus caerulescens, Dysithamnus, Conopophaga, Camptostoma, Capsiempis, Tolmomyias, Colonia (uses tall eucalyptus open canopy a lot), Lathrotriccus, Platyrinchus, Tiaris, Euphonia chlorotica, and Basileuterus flaveolus.

Four forest resident species have increased in number (Pionus, Phaethornis, Arremon, Tachyphonus) in recent years, and one Ramphastos toucan has moved in (above). Nowadays the Pionus uses fruiting trees on the nearby campus, partly explaining greater numbers.

\section{DISCUSSION}

Open-area birds - City and campus to the west, lake clearings within, and cane fields with grassy swales to east and north toward the São José woodlot, provided 33 species near and within the Horto (plus 2 vagrants) and 50 species near São José (where more were checked), for a total of 53 (including the 2 vagrants and Hirundinea, perhaps a vagrant).

Twenty of 33 open-area species rarely or never visit the central lake clearing, which have only 3 reached recently; Pyrocephalus is only a rare winter visitor, so it is not a good place to find open-area birds other than Syrigma, Vanellus, Columba picazuro, Guira, and a few more. Nesting species are few, about 12; even border birds can be rare in the central lake area (below). Cane-field swales off to the east have more birds, and a scattering of grassy-marsh species as well. Rare species present in the Itirapina savannas off to the northwest are more interesting (Willis, 1995). Even the campus and city have more open-area birds, but are being developed rapidly.

Water birds - The Horto lake is an interesting place to show students several water birds, but almost none breed there other than Gallinula, Jacana, and (recently) Agelaius ruficapillus. It is richer in bird life than the dredged and weedremoved Lago Azul in the city center, but subject to occasional cat-tail and water-lily removal (which does stop its developing into a closed marsh). Certain birds (as Porphyrula and Aramus) lose with the cleanup campaigns; others gain. Forty-six 
species (plus 1 vagrant) have been recorded versus 40 in ponds and marshes around the São José woodlot, for a total of 56 (including 1 vagrant).

A major problem with counting numbers of species of these water birds (and some edge birds, see below) is that a given species like the cormorant or egret often uses the lake only one tenth or less of its life. Thus, it spends 0.9 of its time in other areas, which are not counted as "areas" when one says that the Horto has many species of birds compared to a normal forest tract. The duck $D$. viduata certainly spends only half its day in the Horto, using other ponds by day during some months, and is not directly comparable to a swamp bird like Mesembrinibis or Aramides, or a forest bird, that resides all year in the Horto or São José. Even border Caracara only roosts or nests in the Horto, spending the day foraging over open areas, and is not really a "Horto species". Fish-eating and snail-eating water birds, plus ducks, often use distant areas much of the year. Even summer or winter residents, or migrant visitors, use the Horto only part of the year, and could also be counted as "0.5 species" (summer), "0.3 species" (winter) or "0.1-0.2 species" (migrants). If one deducts time spent off the Horto for 21 of 46 of these "C" water birds (minus 0.7 of the year for D. viduata, 0.9 for Phalacrocorax and egrets, 0.5 for Ceryle and Elaenia spectabilis, for instance), some 14 "species" would be deducted from the list, hence only 32 water species and 241 total species would be present. A similar analysis for 16 "part-time" birds of the 40 water birds of São José would subtract 11 species total, leaving 29 water birds "present" (much like the Horto, despite its lake).

As a referee, some years ago, I had to reject a paper claiming that some small city parks with ponds and semi-open zones had more species than did large forested parks in the same region. A major difficulty was that the author recorded few border species in the large park, even though this park obviously had a longer border than the small ones. Obviously, more censuses were done in the forest interior and fewer on the edge in the large park. One has to spend more hours censusing in a large forest tract than in a small one, to get equal hours along the borders. I myself (Willis, 1979, on) have always censused long hours at edges even in large woodlots like those found here.
Edge species - Border species ("B") vary from ones that never enter nearby cane, weeds, bushes, and city, to others like Caracara and Columba cayennensis that roost in but forage mostly outside the woodlots. The sixty-eight border birds in the Horto almost equal the 60 (plus 5 vagrants) in São José. The semiopen lake area and botanic gardens do help these species in the Horto to some extent.

Notably, Ictinia never occur, Accipiter is rare, and Megarynchus vanished recently near São José (perhaps as a result of dry years) but find the botanic garden semi-open woods excellent (forest Parula also disappeared from São José, staying in the canopy and edge of the Horto). However, several species (above) do not reach the lake clearings or have done so only recently, in part due to occasional or careful bush, weed, and tall grass removal near the lake area. Few species use distant or outside areas part-time, except for migrants and summer/winter visitors, so I will not attempt to subtract "part-timers". One does have to avoid problems of reduced observations at edges of large woodlots (see preceding section) which, however, is not a problem here or in São José.

Some cerrado birds appear along the edges occasionally (Colibri, Elaenia obscura, Tyrannus savana, and "vagrant" E. chiriquensis). Cyanocorax cristatellus, Tangara cayana, Piranga, and understory Troglodytes and Coryphospingus of cerrado irregular canopies are common in the taller but similarly irregular-canopy eucalyptus or edges. Motta-Júnior (1990) already showed even greater similarity between cerrado and eucalyptus birds near the campus of the Universidade Federal de São Carlos, where these habitats adjoin.

Certain edge (and forest canopy) species, notably thrushes, emberizids, and hummingbirds, move into the Horto with planted palm (Thraupis palmarum, Tachornis) or fruit trees or flowers (see Antunes, 2000, for use of unusually abundant and constant eucalyptus flowers, possible here only because many species were planted that flower in different months, and commercial cutting every 7 years was not a factor).

Forest birds - 97 forest species (plus 2 vagrants) in the Horto is somewhat less than the 103 (+5) in the much smaller São José woodlot nearby. Obviously, the Horto upper story is generally eucalyptus or of other foreign species, and the dense understory is likely to be botanically depauperate. 
Of 19 migratory species, 10 have not been recorded recently and 3 have declined. A similar decrease in recent years in São José has perhaps been due to dry years, as an unusually strong El Niño occurred in 1982-83 and could have forced southern birds unusually far north (with rains) or temporarily raised their populations. Of 8 summer species, 2 have not been recorded recently and 4 are less common nowadays. Decreases in the Horto could be due to either lumbering or recent dry years

Besides the 12 of 27 migrants and summer species not recorded recently, 12 of 70 resident species have vanished, hence one-quarter of the forest interior avifauna. While Crypturellus tataupa may have been hunted by wandering dogs, and Cathartes killed by hunters, losses of other species seem due to lumbering, drought, or both. Decreases have been registered for 7 of 27 migrant/summer birds and for 29 of 70 residents, nearly $40 \%$ of the avifauna, almost certainly due to logging of large areas. Machado \& Lamas (1996) also registered loss of many species in areas of eucalyptus without native understory.

Three resident understory birds recently increased somewhat, as did an upper-story parrot using campus fruit trees nearby. A toucan from dryer regions of the interior moved in, as did two border species. With rains in 2000-01 after the strong La Niña drought, it may be that some lost or decreasing birds will reinvade or increase in populations. This study started in a wet period and ended with a drought, and the woodlot in the 1990s seeming dryer than in the 1980 s could be related to global warming - one does not know.

One also does not know if logging will continue in the Horto. It could be turned into a productive cellulose or firewood source, with only the small lake and clearing areas used by the public except for jogging and exercise roads in the cutover plantations. Better yet, only the few sapling tracts could be used to make money. (Where the money, gained by logging in 1985-93, went is a mystery.) Other "Hortos" of the old railroad company are being destroyed by the landless poor, when a biologist would have preferred that these areas be subject to environmental impact studies and restored as natural forest reserves, rare in interior São Paulo. The landless poor can always be given cane fields or pastures, though original owners have to be paid (obviously not true when public land is simply given away). It might be interesting to set aside a forest corridor along the Ribeirão Claro, between São José and the Horto, and allow forest birds to move in as the Horto returns to a more natural forest, still leaving old eucalyptus to provide flowers for hummingbirds and without spending money on removal.

Acknowledgments - I thank the Conselho Nacional de Desenvolvimento Científico e Tecnólogico (CNPq) and Universidade Estadual Paulista for support, and students, A. Z. Antunes, and others for help and company in treks. Yoshika Oniki helped with analyses and editing. Publication n. 24 of the Institute for Studies of Nature.

\section{REFERENCES}

ALEIXO, A. \& VIELliARD, J. M. E., 1995, Composição e dinâmica da avifauna da mata de Santa Genebra, Campinas, São Paulo, Brasil. Rev. Brasil. Zool., 12(3): 493-511.

ANTUNES, A. Z., 1996, Levantamento avifaunístico do lago do Horto Florestal "Navarro de Andrade", Rio Claro. Monog. Bach., Biologia, Universidade Estadual Paulista, Rio Claro, 35p.

ANTUNES, A. Z., 2000, Aves que exploram flores de Eucalyptus spp. como recurso: diversidade e ecoetologia. Dissertação de Mestrado, Zoologia, Universidade Estadual Paulista, Rio Claro, SP, 81p.

BENCKE, G. A. \& GRILlO, H. C. Z., 1995, Range expansion of the Pale-breasted Thrush Turdus leucomelas (Aves, Turdidae) in Rio Grande do Sul, Brazil. Iheringia, (79): 175-176.

FERRARI, F. F., 1985, Estudo sobre alguns aspectos do comportamento e da ecologia de galinha-d'água (Gallinula chloropus) e frangos d'água-azuis (Porphyrula martinica) (Aves, Rallidae). Monog. Bach., Biologia, Universidade Estadual Paulista, Rio Claro, 37p.

HÖFLING, E. \& CAMARGO, H. F. de A., 1993, Aves no campus: Cidade Universitária Armando de Salles Oliveira. São Paulo, Inst. Bioc. da USP, 126p.

INGELS, J., ONIKI, Y. \& WILLIS, E. O., 1999, Opportunistic adaptations to man-induced habitat changes by some South American Caprimulgidae. Rev. Brasil. Biol., 59: 563-566.

MACHADO, R. B. \& LAMAS, I. R., 1996, Avifauna associada a um reflorestamento de eucalipto no município de Antônio Dias, Minas Gerais. Ararajuba, 4: 15-22.

MAGAlHÃES, J. C. R. de, 1999, As aves na Fazenda Barreiro Rico. Plêiade, São Paulo, 215p.

MITCHELL, M. H., 1957, Observations on birds of southeastern Brazil. University of Toronto Press, Toronto, $258 \mathrm{p}$. 
MOTTA-JÚNIOR, J. C., 1990, Estrutura trófica e composição das avifaunas de três habitats terrestres na região central do Estado de São Paulo. Ararajuba, 1: 65-71.

ONIKI, Y., WILLIS, E. D. \& WILLIS, M. M., 2002, Citynesting Barred Antshrikes Thamnophilus doliatus (Aves, Thamnophilidae) in São Paulo, Brazil. Rudolstädter nat. hist. schr., Suppl. 4: 85-87.

WILLIS, E. O., 1979, The composition of avian communities in remanescent woodlots in southern Brazil. Pap. Avulsos Zool., S. Paulo, 33: 1-25.

WILLIS, E. O., 1987, Possible long-distance pair migration in Cyanerpes cyaneus. Wilson Bull., 99: 498-499.

WILLIS, E. O., 1991, Expansão geográfica de Netta erythrophthalma, Fluvicola nengeta e outras aves de zonas abertas com a "desertificação" antrópica em São Paulo. Ararajuba, 2: 101-102.

WILLIS, E. O., 1995, Algumas aves de habitats especiais da região de Itirapina (São Paulo). Atualidades Ornitológicas, (68): 7 .
WILLIS, E. O. \& ONIKI, Y., 1987, Invasion of deforested regions of São Paulo state by the picazuro pigeon, Columba picazuro Temminck, 1813. Ciênc. e Cult., 39: 1064-1065.

WILLIS, E. O. \& ONIKI, Y., 1993, New and reconfirmed birds from the state of São Paulo, Brazil, with notes on disappearing species. Bull. Br. Ornithol. Club, 113(1): 23-34.

WILLIS, E. O. \& ONIKI, Y., 2002a, Birds of a central São Paulo woodlot: 1. Censuses 1982-2000. Rev. Brasil. Biol., 62: 197-210.

WILLIS, E. D. \& ONIKI, Y., 2002b, Birds of Santa Teresa, Espírito Santo, Brazil: Do humans add or subtract species? Papéis Avulsos Zool., 12: 193-264. 